\title{
ALTERAÇÕES NA GLICEMIA E NO PERFIL LIPÍDICO PROVOCADAS PELO EXERCÍCIO FÍSICO DURANTE O PROCESSO DE ABSTINÊNCIA ALCOÓLICA EM RATOS WISTAR
}

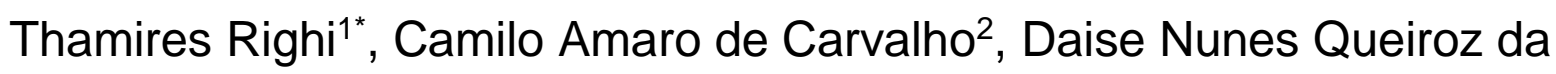
Cunha $^{3}$, Ana Carolina Silva Paiva ${ }^{1}$, Antônio José Natali ${ }^{4}$, Eveline Torres Pereira $^{4}$, Luciana Moreira Lima²

\footnotetext{
1Departamento de Bioquímica e Biologia Molecular da Universidade Federal de Viçosa, MG, Brasil 2 Departamento de Medicina e Enfermagem da Universidade Federal de Viçosa, MG, Brasil 3Departamento de Medicina Veterinária da Universidade Federal de Viçosa, MG, Brasil 4Departamento de Educação Física da Universidade Federal de Viçosa, MG, Brasil *e-mail: thamirighi@gmail.com
}

\section{Resumo}

O etanol afeta todas as células do organismo. Devido aos danos fisiológicos causados pelo seu consumo exagerado, novos modelos de tratamento coadjuvantes têm sido utilizados, dentre eles, a prática da atividade física (AF) aeróbica. Verificamos as alterações provocadas pelo uso de álcool e pela AF no perfil lipídico de ratos Wistar. Foram utilizados 24 animais machos, com 90 dias, divididos em quatro grupos experimentais $(n=6)$ : controle (sem álcool) e sedentário (CS); controle (sem álcool) exercitado (CE); alcoólico e exercitado (AE); e alcoólico sedentário (AS). Foi administrado álcool $(4 \mathrm{~g} / \mathrm{Kg})$ na concentração $20 \% \mathrm{v} / \mathrm{v}$ por 4 semanas. Ao final da $4^{\mathrm{a}}$ semana iniciou-se o programa de AF em esteira com os grupos AE e CE. Os animais correram 5 dias/semana, 1hora/dia, por 2 semanas, na intensidade de $65 \%$ da velocidade média comum. A eutanásia foi feita pela anestesia com isoflurano e retirada total do sangue por punção cardíaca. O colesterol total (CT) (mg/dL), triglicerídeos (TG) $(\mathrm{mg} / \mathrm{dL})$ e glicose $(\mathrm{GL})(\mathrm{mg} / \mathrm{dL})$ foram determinados por métodos enzimático-colorimétricos específicos. Utilizamos ANOVA seguido de teste de Tukey para todos os parâmetros devido à distribuição normal e homocedasticidade, $\mathrm{p}<0,05$ e o teste de correlação de Pearson para verificar as correlações lineares entre as variáveis. A GL foi significativamente mais baixa nos grupos CE $(118 \pm 7)$ e AS $(124 \pm 15)$ quando comparada aos grupos CS 
$(161 \pm 12)$ e $\mathrm{AE}(150 \pm 18)$. O CT total apresentou-se mais elevado nos grupos $\mathrm{CE}(57 \pm 6)$ e AS $(56 \pm 10)$ quando comparados com o grupo AE (45 \pm 3$)$. Os TL foram mais elevados no grupo AS $(171 \pm 23)$ quando comparado com os grupos CS $(125 \pm 31)$ e AE $(110 \pm 15)$. Não foram observadas diferenças significativas entre os quatro grupos para o HDL. Concluímos que a associação do álcool e da AF modificaram significativamente os níveis de GL e CT dos ratos Wistar quando comparados com grupos alcóolico e sedentário.

Palavra-chave: perfil lipídico, álcool, atividade física em associação.

Apoio financeiro: Proext 